\title{
Impact of mode-hopping noise on InGaN edge emitting laser relative intensity noise properties
}

\author{
Antoine Congar, Kamal Hussain, Christelle Pareige, Raphaël Butté, Nicolas Grandjean, \\ Pascal Besnard and Stéphane Trebaol
}

\begin{abstract}
In this study we report, through a comparative analysis of static and dynamic measurements, on the impact of mode hopping on the overall intensity noise dynamics of InGaN edge emitting lasers. Mode clustering usually observed in InGaN lasers enhances the mode competition and then the laser intensity fluctuation. Variations as large as $20 \mathrm{~dB}$ have been observed. The coexistence of longitudinal modes induces mode competition and then unstable bimodal lasing. This mode competition is mediated by temporal fluctuations of spontaneous emission through nonlinear cross saturation of the optical gain. The subsequent enhancement of the relative intensity noise can be detrimental for applications where the intensity stability of InGaN lasers is a key parameter. Our experimental observations are supported by a simple rate equation model. We point out the major importance of the different modal gain in the bimodal regime on the overall intensity noise of the laser.
\end{abstract}

Index Terms-Relative intensity noise (RIN), nonlinear gain, Semiconductor GaN lasers.

\section{INTRODUCTION}

$\mathbf{S}$ INCE the first demonstration of commercially available GaN based laser diodes in 1997 [1], blue emitting coherent light sources have opened up new opportunities in areas such as optical data storage [2], visible light communication [3]-[5] (VLC), linear and nonlinear spectroscopy for fundamental [6], [7] and industrial [8] applications, pico-projection [9] and lighting [10]. Without being exhaustive, this list highlights the benefits offered by $\mathrm{GaN}$ lasers.

In the aforementioned applications, the stability of the laser intensity is one of the key parameters. Indeed, for applications that use modulation in the $\mathrm{MHz}$ to $\mathrm{GHz}$ range, like data storage, VLC and pico-projection, the intensity noise has to be calibrated on the targeted bandwidth. In the same vein, for spectroscopic applications for which the signal to noise ratio is the relevant parameter, the noise of the laser is the limiting parameter and has to be quantified. A deep understanding of the dynamics of InGaN lasers is mandatory since it affects the overall characteristics of the devices in which they might be embedded.

In lasers, the noise response is directly governed by the photon and the electron populations dynamics. In particular in monolithic semiconductor lasers, the two population dynamics are coupled and energy exchange occurs at a frequency called

Antoine Congar, Kamal Hussain, Christelle Pareige, Pascal Besnard and Stéphane Trebaol are with the UMR FOTON, CNRS 6082, Université de Rennes 1, Enssat, F22305, Lannion, France

Raphaël Butté and Nicolas Grandjean are with the Institute of Physics, Ecole Polytechnique Fédérale de Lausanne (EPFL), CH-1015 Lausanne, Switzerland the relaxation frequency. This type of lasers is called Class B lasers in the Arecchi classification [11]. Intensity noise is one of the observables allowing to better understand the impact of the structure on the laser dynamics. Indeed, both the cavity design (photon population) and the epitaxial structure (electron population) directly impact the noise dynamics. Correlations between intensity noise and spectral dynamics have been widely studied for infrared lasers operating in the telecom bands [12], [13] and noise classification based on their origin is well established [14].

During the last decade, studies on the spectral dynamics of blue laser diodes contributed to the understanding of the manifestation of mode clustering [15]-[19]. In particular, Weig et al. studied multimode hopping occurring in blue laser diodes through time-resolved measurements [20] showing the role of the nonlinear gain term on the spectral dynamics.

Despite their importance, the correlations existing between the optical spectrum structure and intensity fluctuations mediated by noise in blue emitting laser diodes have not been addressed yet.

In this paper, we study the impact of the modal structure on the intensity dynamics of InGaN edge emitting laser diodes. Simultaneous measurements of the optical spectrum and relative intensity noise (RIN) as a function of pump current give access to the rich dynamics of those lasers. In particular, we show that for specific pump current operations, the laser can display a variety of lasing regimes, each of them featuring their own intensity noise signature. Actually, lasing dynamics can be classified in two regimes. The laser diode behaves either in single mode operation with usually a very low noise level or in multimode operation where two or more modes lase in a stable or unstable manner.

This paper aims at describing and analyzing these two regimes in terms of noise dynamics. It is organized as follows. First, we recall the main characteristics of the structure under test and describe the experimental optical setup. Then we detail and discuss our experimental observations. Simulation results based on nonlinear coupled rate equations support our observations and allow us to point out the key role of gain imbalance and the nonlinear cross-saturation term of the gain on the RIN in multimode operation.

\section{EXPERIMENTAL SETUP}

The laser structure was grown by metalorganic vapor phase epitaxy on a $c$-plane free-standing $\mathrm{GaN}$ substrate. The active region consists of two $4.5 \mathrm{~nm}$ wide $\mathrm{InGaN}$ quantum wells 

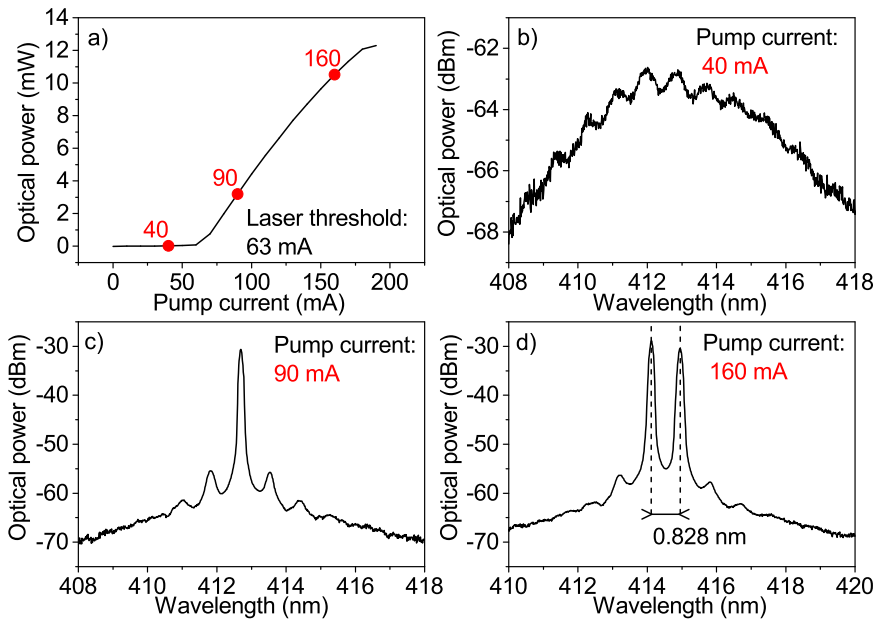

Fig. 1. (Color online) a) L-I curve of the laser. Optical spectra of the diode b) below $(I=40 \mathrm{~mA}), \mathrm{c})$ above $(I=90 \mathrm{~mA})$ and $\mathrm{d})$ far above $(I=160 \mathrm{~mA})$ the laser threshold $\left(I_{t h}=63 \mathrm{~mA}\right)$.

separated by $12 \mathrm{~nm}$ undoped $\mathrm{In}_{0.05} \mathrm{Ga}_{0.95} \mathrm{~N}$ barriers with low In-content and a $p$-AlGaN electron blocking layer. The bottom and top cladding are $1 \mu \mathrm{m}$ and $500 \mathrm{~nm}$ in thickness, respectively. The laser consists of a $680 \mu \mathrm{m}$ long ridge structure. The width of the ridge is limited to $2.5 \mu \mathrm{m}$ to ensure single mode transverse operation. A five-layer dielectric distributed Bragg reflector $\left(\mathrm{SiO}_{2} / \mathrm{Ta}_{2} \mathrm{O}_{5}\right)$ with a reflectivity of $\sim 95 \%$ is deposited on one of the facets to maximize the directivity of the emission on the opposite facet. The structure is described in more details elsewhere [21]. The laser is characterized using an optical setup allowing to study RIN, L-I curves and optical spectra together. These simultaneous measurements permit to analyze the static and dynamical behavior of the laser. The L-I curve and the RIN are collected through a silicon photodiode followed by a $20 \mathrm{~dB}$ low noise RF amplifier. The overall detection scheme has a $2 \mathrm{GHz}$ bandwidth. The RIN measurements are obtained following a method described elsewhere [22]. The spectrometer has a resolution of $120 \mathrm{pm}$ which is sufficient to resolve the spectral dynamics of the laser under study.

\section{EXPERIMENTAL RESULTS}

\section{A. Static measurements}

First, we study the static response of the laser based on L-I curve measurements. The results are presented in Figure 1 a). Above the laser threshold $\left(I_{\mathrm{th}}=63 \mathrm{~mA}\right)$, the optical power has a linear dependency to the pump current masking the dynamics of the spectral energy redistribution. Indeed, Figures $1 \mathrm{~b}$ ) to $1 \mathrm{~d}$ ) show the optical spectrum dynamics for different pump currents. Figure $1 \mathrm{~b}$ ) displays the optical spectrum below threshold ( $I=40 \mathrm{~mA}$ ) corresponding to the amplified spontaneous emission regime. It is important to note that the spacing between the resonances of the Airy function of the cavity is expected to be $36 \mathrm{pm}$ at $415 \mathrm{~nm}$. Nevertheless a strong modulation of the gain curve (Fig. $1 \mathrm{~b}$ )) is observed. The gain exhibits a ripple with $\sim 800$ pm periodicity. As shown in Figures $1 \mathrm{c}$ ) and $1 \mathrm{~d}$ ), this ripple determines the operating longitudinal modes. This discrepancy between experimentally measured and theoretically predicted free spectral range in GaN laser diodes is commonly observed [23]-[26]. In the literature, different origins can be attributed to such mode clustering, in particular cracks in the GaN structure and substrate mode coupling. Indeed, due to aging of the structure, microcracks can induce subcavities introducing a modulation of the gain [6]. The crack position is, by nature, randomly distributed and should not lead to a periodic modulation of the cavity modes. Instead, the periodic modulation of the gain, that subsequently allows only a few modes to lase, is attributed to coupling between the longitudinal modes and substrate modes. As reported by Laino et al. [16] the thickness and the optical index of the $n$-cladding layer play a major role on the modal gain modulation. Thicknesses below $2 \mu \mathrm{m}$ allow the coupling between longitudinal modes and substrate modes giving rise to gain ripple (Fig. 1 b)). Hence, the periodicity of the gain ripple is determined by the length of the ridge and the bottom cladding layer thickness. Nevertheless, the origin of the mode clustering is not primordial for our study.

When increasing the pump current above threshold $(I=$ $90 \mathrm{~mA}$ ), the laser displays one cluster whose magnitude is lying $23 \mathrm{~dB}$ above the others. One cluster includes less than ten successive longitudinal operating modes. Increasing the pump current up to $160 \mathrm{~mA}\left(2.54 I_{\text {th }}\right)$ the laser behaves in bicluster operation and displays two 828 pm spaced clusters. In the following, we study simultaneously the overall intensity fluctuations of the laser and its spectral structure.

\section{B. Spectral and RIN measurements}

We record continuously the optical spectrum and the RIN as a function of pump current above threshold. Figure 2 a) shows the spectrum distribution and Figure $2 \mathrm{~b}$ ) the RIN. Our first observation concerns the redshift of lasing clusters (Fig. 2 a)). Two trends can be extracted. First, there is a redshift of the gain profile due to a temperature increase mediated by the current density [17], [27]. This phenomenon is at the origin of the laser swinging between single and bicluster operation. Second, a tiny variation in the refractive index due to a change in the carrier density will also redshift the clusters [28]. These two effects contribute to the redshift of the lasing wavelengths with two specific slopes. We measure for the gain and the clusters shifts of $23.9 \mathrm{pm} / \mathrm{mA}$ and 7.5 $\mathrm{pm} / \mathrm{mA}$, respectively. The related ratio of 3.2 is comparable to previously reported values [25], [28]. A ratio larger than one allows adjacent clusters to lase for some pump current values since the gain curve redshifts faster than the clusters do.

A comparative study of Figure 2 a) with Figure 2 b) allows to identify the impact of the lasing regime on RIN properties. In general, the RIN curve of a laser diode can be analyzed considering three frequency windows. At low frequency $(<1 \mathrm{MHz})$ flicker noise mainly due to carrier injection dominates. At intermediate frequencies, from $1 \mathrm{MHz}$ to few $\mathrm{GHz}, \mathrm{RIN}$ curves display a quite flat plateau mostly related to spontaneous emission. At higher frequencies, relaxation oscillations specific to class B lasers can be observed. Our 

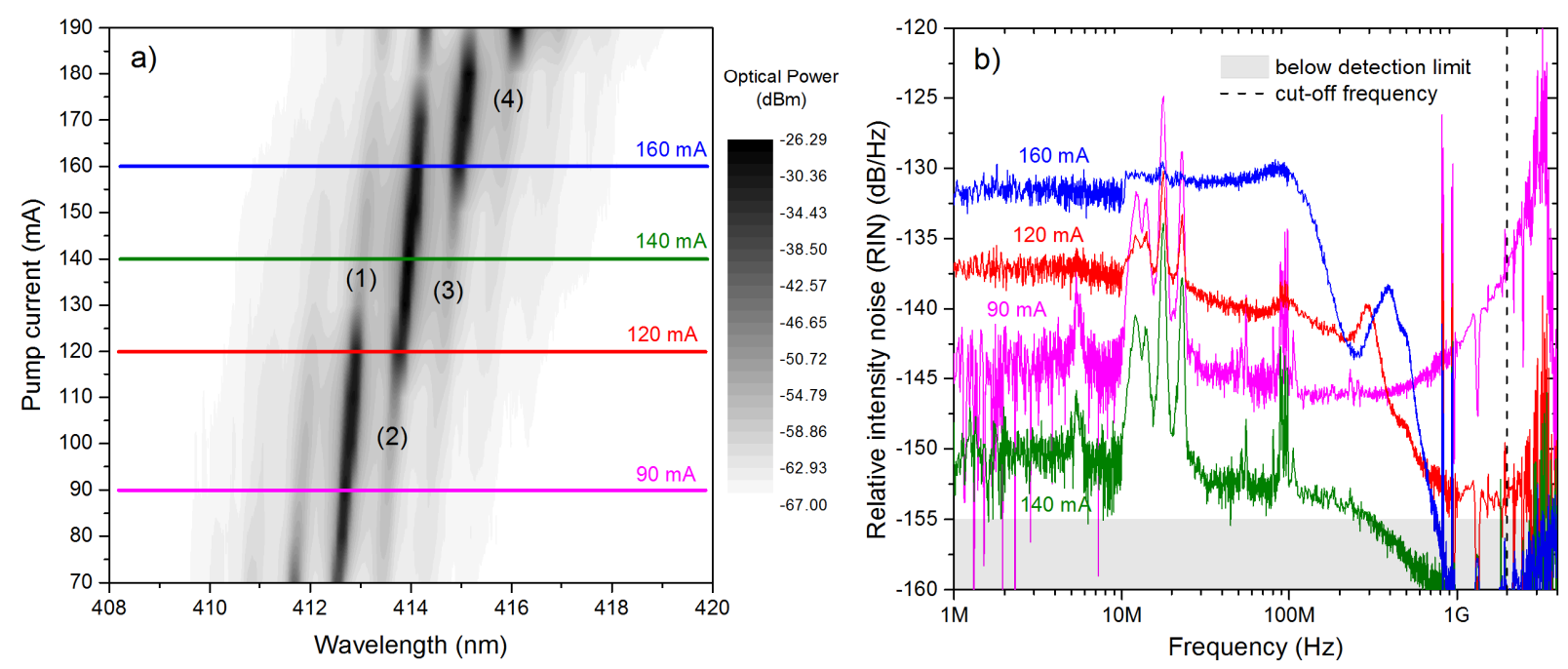

Fig. 2. (color online) a) Optical spectrum evolution as a function of pump current above the laser threshold. b) RIN measurements for specific current values revealing the impact of mode hopping competition in InGaN laser diodes. The strong peaks visible between 10 and $25 \mathrm{MHz}$ and at $100 \mathrm{MHz}$ and $1 \mathrm{GHz}$ are artefacts from electromagnetic perturbations of the setup.

study focuses on the intermediate frequency range from 1 $\mathrm{MHz}$ to $1 \mathrm{GHz}$.

As previously mentioned, slightly above the laser threshold $[I=90 \mathrm{~mA}]$, the laser displays a single cluster [mode 1 in Fig. 2 a)]. The corresponding RIN measurement is presented in Figure $2 \mathrm{~b}$ ), where the strong peaks visible between 10 and $25 \mathrm{MHz}$ and at $100 \mathrm{MHz}$ and $1 \mathrm{GHz}$ are artefacts from electromagnetic perturbations of the setup. The RIN behavior is specific to that of a class B laser in single mode operation with constant low level around $-145 \mathrm{~dB} / \mathrm{Hz}$ at intermediate frequencies $[1-1000] \mathrm{MHz}$ and a rise at frequencies above $1 \mathrm{GHz}$, which is a signature of relaxation oscillations. For a pump current corresponding to twice the threshold $[I=120 \mathrm{~mA}]$, the spectrum displays two clusters (1 and 2), featuring an enhancement of the RIN amplitude at low Fourier frequencies [1 - 300] $\mathrm{MHz}$ (Fig. 2 b)). For increasing currents $[I=140 \mathrm{~mA}]$, the smooth redshift of the gain favors cluster 2 with respect to cluster $\mathbf{1}$. The laser then follows the behavior of a single mode laser characterized by a RIN amplitude below $-150 \mathrm{~dB} / \mathrm{Hz}$ [Fig. $2 \mathrm{~b}$ )]. For $I$ reaching $160 \mathrm{~mA}\left[2.54 I_{\mathrm{th}}\right]$, the continuous shift of the gain curve allows again bicluster operation to occur [clusters 2 and 3]. RIN enhancement as large as $20 \mathrm{~dB}$ with respect to stable single cluster operation is measured at intermediate frequencies [1 - 400] MHz. The RIN curve features a strong resonance around $300 \mathrm{MHz}$ when two clusters of modes are present.

We summarize the overall dynamics of the laser in Figure 3. The RIN level (Figure 3 a)) at an intermediate frequency (30 $\mathrm{MHz}$ ) corresponding to the amplitude of each lasing mode (Figure $3 \mathrm{~b}$ )) is shown as a function of pump current. As already explained when single mode operation occurs, the RIN amplitude reaches its minimum (below $-140 \mathrm{~dB} / \mathrm{Hz}$ ). For unstable bimodal operation, the RIN increases reaching a peak when the amplitude of both lasing modes is maximum $[I=120 ; 160 \mathrm{~mA}]$.

Although we cannot precisely determine the number of consecutive longitudinal modes within one cluster we make the hypothesis that the behavior of the laser can be understood using a simple single/bimodal laser approach. Model simulations will corroborate this hypothesis. In bimodal lasing operation, two successive modes having similar intensity amplitude can compete to drain the gain through stimulated emission. These two modes, having approximately the same amplitude, have difficulties to equally share the available gain contributing to intensity fluctuations and even mode switching. We attribute the RIN resurgence at intermediate frequencies to mode hopping due to temporal fluctuations of the spontaneous emission. The spontaneous noise is enhanced through the optical gain by self- and crosssaturation terms [29]. Indeed, this intermediate frequency intensity noise follows a Lorentzian profile with a plateau from $1 \mathrm{MHz}$ to $100 \mathrm{MHz}$ and a cut-off frequency around 100 $\mathrm{MHz}$. This mode competition phenomenon is triggered by the spontaneous emission, which is a stochastic process described by a Poisson distribution [30] that explains the white noise shape.

The appearance of a resonance around $300 \mathrm{MHz}$ shows that modes exchange energy preferentially around this hopping frequency [31], [32]. The hopping resonance observed in the bimodal regime reflects a partial antiphase noise since this contribution is visible for the total intensity noise. This partial antiphase noise has been already reported in other types of semiconductor lasers [33], [34]. A coherent antiphase noise would have been only observed on each mode characterized separately. This resonance behavior has been reported through time measurements by Weig et al. [20] in the case of GaN laser diodes. 

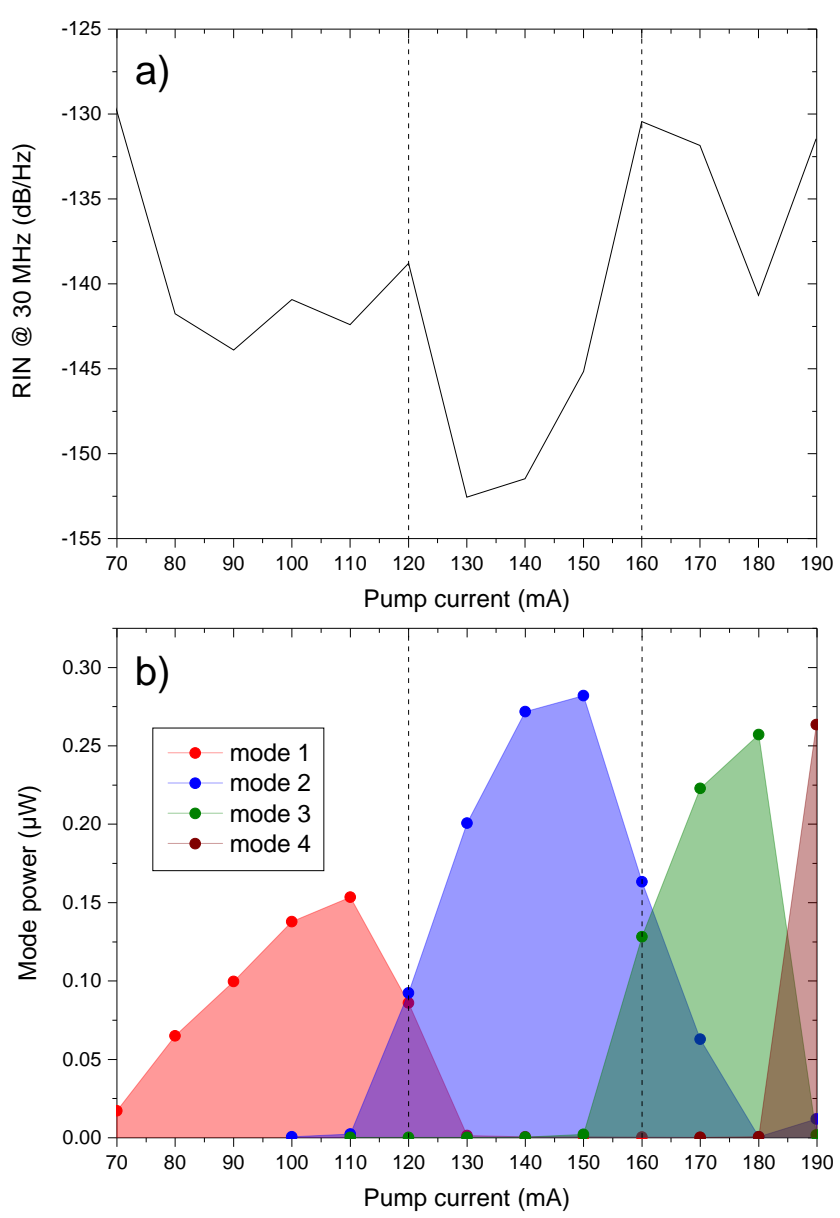

Fig. 3. (Color online) a) RIN of the laser at $30 \mathrm{MHz}$ and b) integrated power of each mode numbered from 1 to 4 . Both figures are plotted as a function of pump current.

\section{ANALytical Model}

To confirm our hypothesis and assess our experimental observations, we model the operation of a semiconductor laser diode around $415 \mathrm{~nm}$ featuring two $800 \mathrm{pm}$ spaced longitudinal modes coupled through nonlinear gain terms and derive the RIN in such a case. In particular, we will show that gain imbalance and the nonlinear cross-saturation term are the physical parameters at the origin of the RIN resurgence in bicluster operation.

The rate equations for such bimodal operation are given by [35],

$$
\begin{array}{r}
\frac{d P_{1}(t)}{d t}=\left(G_{1}-\gamma_{1}\right) P_{1}(t)+R_{s p}+F_{p}(t), \\
\frac{d P_{2}(t)}{d t}=\left(G_{2}-\gamma_{2}\right) P_{2}(t)+R_{s p}+F_{p}(t), \\
\frac{d N(t)}{d t}=\frac{I}{q}-\frac{N(t)}{\tau_{s}}-\sum_{k=1}^{2} G_{k} P_{k}(t)+F_{N}(t),
\end{array}
$$

where $P_{k}$ are the photon numbers in the cavity, $k$ indicates the mode number, $G_{k}$ and $\gamma_{k}$ are the gain and loss of the $k^{t h}$ mode, respectively. $N$ is the carrier number, $I$ is the pump current, $q$ represents the charge of the electron and $\tau_{s}$ the carrier lifetime. $R_{s p}$ is the spontaneously emitted photon rate and $F_{p}$ and $F_{N}$ are the Langevin noise source terms with zero mean and unit standard deviation as defined in the Appendix. We used the small signal approximation to linearize Eqs. 1. $\delta N, \delta P_{1}$ and $\delta P_{2}$ indicate the small variations of carrier number and photon number of each mode. The modal gains have nonlinear components so that [35],

$$
\begin{aligned}
& G_{1}=\bar{G}_{1}+G_{N} \delta N-B \delta P_{1}-D \delta P_{2}, \\
& G_{2}=\bar{G}_{2}+G_{N} \delta N-B \delta P_{2}-D \delta P_{1},
\end{aligned}
$$

where $\bar{G}_{1}$ and $\bar{G}_{2}$ are the steady-state gains of the two modes, $G_{N}$ is the differential gain coefficient. The two modes are coupled through the gain introducing nonlinear contributions from $B$, the self-saturation term, and $D$, which is the crosssaturation term. The latter determines the strength of the coupling between the modes. The saturation terms are approximately given by [29],

$$
\begin{gathered}
B=\frac{9}{4} \frac{\Gamma G_{N} R_{c v}^{2}}{\epsilon_{0} n_{r}^{2}} \frac{\hbar \omega}{V}\left(\frac{\tau_{i n}}{\hbar}\right)^{2}\left(N_{t h}-N_{s}\right), \\
D=(4 / 3) B
\end{gathered}
$$

where $\Gamma$ is the confinement factor of the light in the active region, $R_{c v}$ is the dipole moment, $V$ is the volume of the active region, $n_{r}$ is the refractive index of the active region, $\tau_{i n}$ is the intraband relaxation time, $N_{t h}$ is the carrier number at threshold and $N_{s}\left(\sim 0.8 N_{t h}\right)$ is the carrier saturation coefficient. Fourier transform of linearized versions of Eqs. 1 gives access to the small signal variations of the two considered modes, namely $\delta \tilde{P}_{1}(\omega)$ and $\delta \tilde{P}_{2}(\omega)$.

Recalling that the RIN of the dual-mode is given by,

$$
R I N(\omega)=\lim _{T \rightarrow \infty} \frac{1}{T} \frac{\left|\delta \tilde{P}_{1}(\omega)+\delta \tilde{P}_{2}(\omega)\right|^{2}}{\left(\bar{P}_{1}+\bar{P}_{2}\right)^{2}},
$$

and replacing the expression of $\delta \tilde{P}_{1}(\omega)$ and $\delta \tilde{P}_{2}(\omega)$ in Eq. (4) gives,

$$
\begin{aligned}
R I N(\omega)= & \frac{2 D_{11}\left|T_{F_{1}}\right|^{2}+2 D_{22}\left|T_{F_{2}}\right|^{2}+2 D_{1 N}\left|T_{F_{1}}^{*} T_{F_{N}}\right|}{\left|T_{Z}\right|^{2}\left(\bar{P}_{1}+\bar{P}_{2}\right)^{2}} \\
& +\frac{2 D_{2 N}\left|T_{F_{2}}^{*} T_{F_{N}}\right|+2 D_{N N}\left|T_{F_{N}}\right|^{2}}{\left|T_{Z}\right|^{2}\left(\bar{P}_{1}+\bar{P}_{2}\right)^{2}}
\end{aligned}
$$

where

$$
\begin{gathered}
T_{F_{1}}=j \omega+\frac{R_{s p}}{\bar{P}_{2}}-(D-B) \bar{P}_{2}-\frac{\left(\bar{G}_{1}-\bar{G}_{2}\right) G_{N} \bar{P}_{2}}{\Gamma_{N}+j \omega}, \\
T_{F_{2}}=j \omega+\frac{R_{s p}}{\bar{P}_{1}}-(D-B) \bar{P}_{1}+\frac{\left(\bar{G}_{1}-\bar{G}_{2}\right) G_{N} \bar{P}_{1}}{\Gamma_{N}+j \omega}, \\
T_{F_{N}}=\frac{G_{N}}{\Gamma_{N}+j \omega}\left[j \omega\left(\bar{P}_{1}+\bar{P}_{2}\right)\right. \\
\left.+R_{s p}\left(\frac{\bar{P}_{1}}{\bar{P}_{2}}+\frac{\bar{P}_{2}}{\bar{P}_{1}}\right)-2(D-B) \bar{P}_{1} \bar{P}_{2}\right],
\end{gathered}
$$




$$
\begin{aligned}
& T_{Z}=-\omega^{2}+j \omega R_{s p}\left(\frac{1}{\bar{P}_{1}}+\frac{1}{\bar{P}_{2}}\right)+j \omega B\left(\bar{P}_{1}+\bar{P}_{2}\right) \\
& +\frac{j \omega G_{N}}{\Gamma_{N}+j \omega}\left(\bar{G}_{1} \bar{P}_{1}+\bar{G}_{2} \bar{P}_{2}\right)+\frac{G_{N} R_{s p}}{\Gamma_{N}+j \omega}\left(\frac{\bar{G}_{1} \bar{P}_{1}}{\bar{P}_{2}}+\frac{\bar{G}_{2} \bar{P}_{2}}{\bar{P}_{1}}\right) \\
& -\frac{G_{N}\left(\bar{G}_{1}+\bar{G}_{2}\right)(D-B) \bar{P}_{1} \bar{P}_{2}}{\Gamma_{N}+j \omega}+\frac{R_{s p}^{2}}{\bar{P}_{1} \bar{P}_{2}} \\
& +B R_{s p}\left(\frac{\bar{P}_{1}}{\bar{P}_{2}}+\frac{\bar{P}_{2}}{\bar{P}_{1}}\right)-\left(D^{2}-B^{2}\right) \bar{P}_{1} \bar{P}_{2} .
\end{aligned}
$$

where diffusion coefficients $D_{i j}$ are defined as in [35]. In the following, we show the impact of gain imbalance on the RIN amplitude (IV-A). In section (IV-B), the link between an increase of the cross nonlinear term strength and the RIN resurgence bandwidth is detailed. Finally, we use the model to reproduce our experimental observations (IV-C).

\section{A. Effect of the gain imbalance on the RIN}

In this section we aim to study the impact of gain imbalance on the total RIN amplitude. To do so, we plot in Figure 4 the RIN as a function of gain ratio $\Delta=\bar{G}_{2} / \bar{G}_{1}$. We fix the value of the nonlinear gain terms considering the difference as $D-B=400 \mathrm{~s}^{-1}$. The pump current is fixed to $1.9 I_{t h}$ and the power ratio $r=P_{1} / P_{2}$ is equal to 1 . When $\Delta=1$, the noise of the bimodal laser is minimal and corresponds to the RIN behavior of a stable bimodal laser. In such a case, fluctuations in both modes cancel out since their amplitude is exactly equal. On the other hand, when $\Delta$ is different from 1 there is a sudden jump in the low frequency RIN of the dual-mode. This can be explained from Eq. (5) considering a special case. We assume that $\bar{P}_{1}=\bar{P}_{2}=\bar{P}$ and derive the expression of the RIN for $\omega=0$. According to Eqs. 3, we thus have $D=B=0$. The RIN at low frequency is then given by

$$
\begin{aligned}
& \operatorname{RIN}(\omega=0)= \\
& \frac{R_{s p}\left(\frac{1}{\bar{P}}-\frac{2 G_{N}}{\Gamma_{N}}\right)+2\left(\frac{G_{N}}{\Gamma_{N}}\right)^{2}\left(2 R_{s p} \bar{P}+\bar{N} / \tau_{s}\right)}{\left[\frac{R_{s p}}{P}+\frac{G_{N} \bar{P}}{\Gamma_{N}}\left(\bar{G}_{1}+\bar{G}_{2}\right)\right]^{2}} \\
& +\frac{\left(\frac{G_{N} \bar{P}}{\Gamma_{N} R_{s p}}\right)^{2}\left(\bar{G}_{1}-\bar{G}_{2}\right)^{2} R_{s p} \bar{P}}{\left[\frac{R_{s p}}{P}+\frac{G_{N} \bar{P}}{\Gamma_{N}}\left(\bar{G}_{1}+\bar{G}_{2}\right)\right]^{2}} .
\end{aligned}
$$

From the last term in Eq. (7), we see that amplitude RIN resurgence in the low frequency range is due to a different amplification of the spontaneous emission in both modes. Noise terms in the two modes cancel each other only when $\bar{G}_{1}=\bar{G}_{2}$. As the difference between modal gains increases, the noise fluctuation in one mode becomes higher than in the other mode. As a result the dual-mode is impacted by a noise component at low frequency explaining the sudden jump in the low frequency RIN curve for $\Delta<1$.

\section{B. Effect of the nonlinear cross-saturation term on the RIN}

We now fix the gain ratio to an arbitrary value (here, $\Delta=0.8$ ) and vary the nonlinear terms amplitudes to study

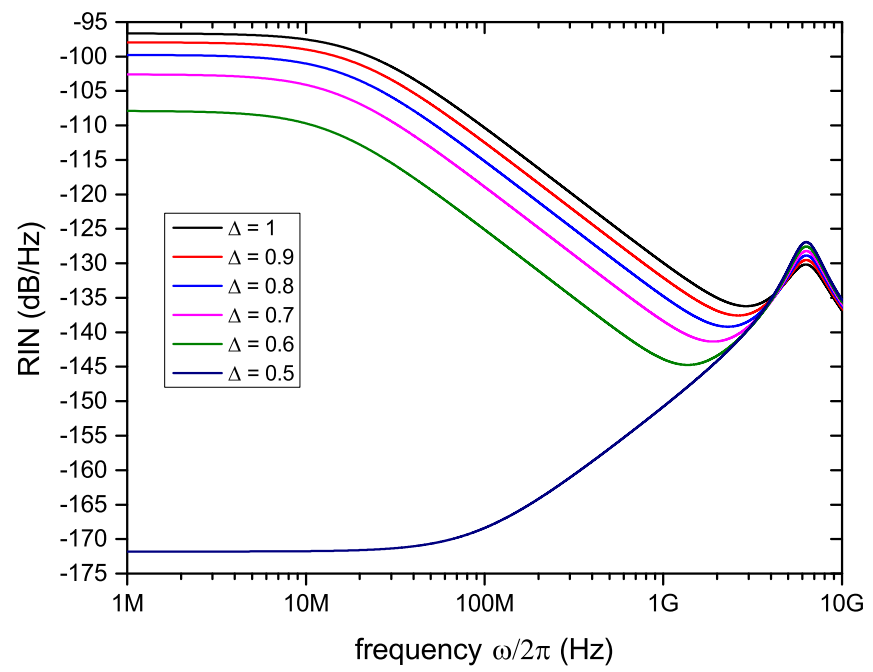

Fig. 4. Variation of the dual-mode RIN for different values of $\Delta=\bar{G}_{2} / \bar{G}_{1}$. Here $D-B=400 \mathrm{~s}^{-1}, r=1$ and $I=1.9 I_{t h}$.

their impact on the total RIN(the pump curent and the power ratio remain the same as in A). Figure 5 shows that as $D-B$ is increased the cut-off frequency $\Omega_{C}$ increases to higher values. This phenomenon reveals that the strength of the coupling between the two modes is related to an increase in the cross saturation-term amplitude. A larger coupling strength increases the rate at which modes exchange energy. The impact on the RIN is an increase in the bandwidth for which spontaneous emission is amplified through the gain.

In our laser, the origin of the cross-saturation term might be attributed to spectral hole burning [36]. Due to the large spectral detuning between the two modes, $800 \mathrm{pm}$ (corresponding to a beating period of $0.72 \mathrm{ps}$ ), spectral hole burning is not mediated by the population lifetime $(\approx$ ns $)$ but by the intrinsic relaxation lifetime $(\approx$ hundreds of $\mathrm{fs})$.

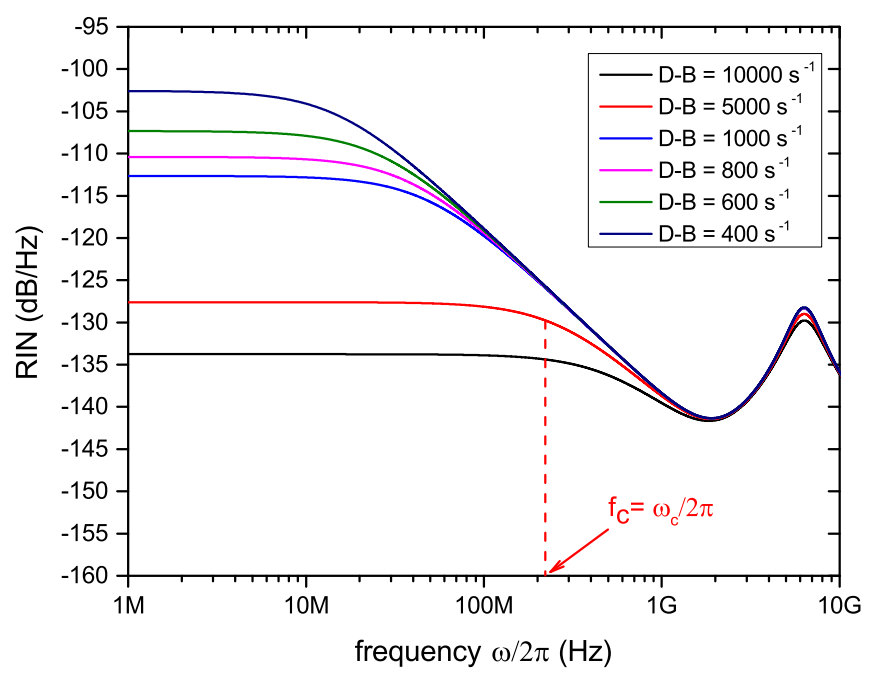

Fig. 5. Variation of the dual-mode RIN for different values of $D-B$. Here $\bar{G}_{2}=0.8 \bar{G}_{1}, r=1$ and $I=1.9 I_{t h}$. 
TABLE I

BLUE LASER PARAMETERS USED FOR THE ANALYTICAL MODEL. NON-REFERENCED PARAMETERS ARE EXTRACTED FROM THE CHARACTERIZATION OF THE LASER DIODE

\begin{tabular}{ll}
\hline Parameter & Value \\
\hline Carrier lifetime $\left(\tau_{s}\right)$ & $2.43 \mathrm{~ns}[37]$ \\
Carrier number at threshold $\left(N_{t h}\right)$ & $7.65 \times 10^{8}$ \\
Rate of spontaneous emission $\left(R_{s p}\right)$ & $1.573 \times 10^{13} \mathrm{~s}^{-1}$ \\
Decay rate of the mode photons $(\gamma)$ & $3.52 \times 10^{11} \mathrm{~s}^{-1}$ \\
Steady-state gain $\left(\bar{G}_{1}\right)$ & $6.79 \times 10^{11} \mathrm{~s}^{-1}$ \\
Gain coefficient $\left(G_{N}\right)$ & $3.55 \times 10^{3} \mathrm{~s}^{-1}$ \\
Dipole moment $\left(R_{c v}^{2}\right)$ & $1.8 \times 10^{-57} \mathrm{C}^{2} \mathrm{~m}^{2} \quad[20]$ \\
Refractive index of the active region $\left(n_{r}\right)$ & $2.53[17]$ \\
Intraband relaxation time $\left(\tau_{i n}\right)$ & $80 \mathrm{fs}$ \\
Group refractive index $\left(n_{g}\right)$ & 3.48 \\
Confinement factor $(I)$ & 0.043 \\
Active region volume $(V)$ & $30 \mu \mathrm{m}$ \\
Threshold current $\left(I_{t h}\right)$ & $63 \mathrm{~mA}$ \\
\hline
\end{tabular}

\section{Comparison to experimental results}

We then use this analytical model to reproduce our experimental observations. Experimental RIN curves of the laser are reproduced analytically for various combinations of pump current $I$ and intensity ratio $r$ in Figure 6 . The values of the parameters used in the model are summarized in Table I. We only have two adjustable parameters in our model $\tau_{\text {in }}$ and $\Delta$. Non-referenced parameters are calculated using values extracted from experimental characterizations. We determine the $\tau_{i n}$ value by looking for the same cut-off frequency than the one deduced from experiment, i.e. around $100 \mathrm{MHz}$. The value of $\tau_{i n}$ that we use is of the same order than values found in the literature [20]. Considering $\tau_{i n}=80 \mathrm{fs}$ in Eq. (4) we obtain $B \sim 3 \times 10^{4} \mathrm{~s}^{-1}$ and $D \sim 4 \times 10^{4} \mathrm{~s}^{-1}$ giving a difference of strength for the nonlinear terms $D-B=$ $1 \times 10^{4} \mathrm{~s}^{-1}$. The gain ratio $\Delta$ is determined by looking for a RIN amplitude (at $1 \mathrm{MHz}$ for $I=120 \mathrm{~mA}$ ) of -136 $\mathrm{dB} / \mathrm{Hz}$, leading to $\Delta=0.87$. This gain imbalance between two consecutive clusters is justified by the large spectral cluster spacing of $828 \mathrm{pm}$. Under this condition, the model reproduces the experimental observations of Figure $2 b$ ) in particular the relaxation frequency shift, the RIN resurgence in bimodal operation and the cut-off frequency at $100 \mathrm{MHz}$. Amplitude discrepancy of the RIN for $I=90 \mathrm{~mA}$ and $140 \mathrm{~mA}$ is related to the limited sensitivity of the experimental setup. The antiphase noise resonance appearing around $300 \mathrm{MHz}$ is not included in our simple model based on a perturbative approach. Numerical simulations would have to be performed to reproduce this behavior.

\section{CONCLUSION}

In conclusion, we have shown that mode clustering strongly impacts the intensity stability of InGaN laser diodes. Strong resurgence of the RIN in bicluster operation or single mode RIN dynamics in single cluster operation is observed. Using a bimodal rate equation model, we were able to reproduce our experimental observations and to highlight the main role of gain imbalance and the nonlinear cross-saturation gain term

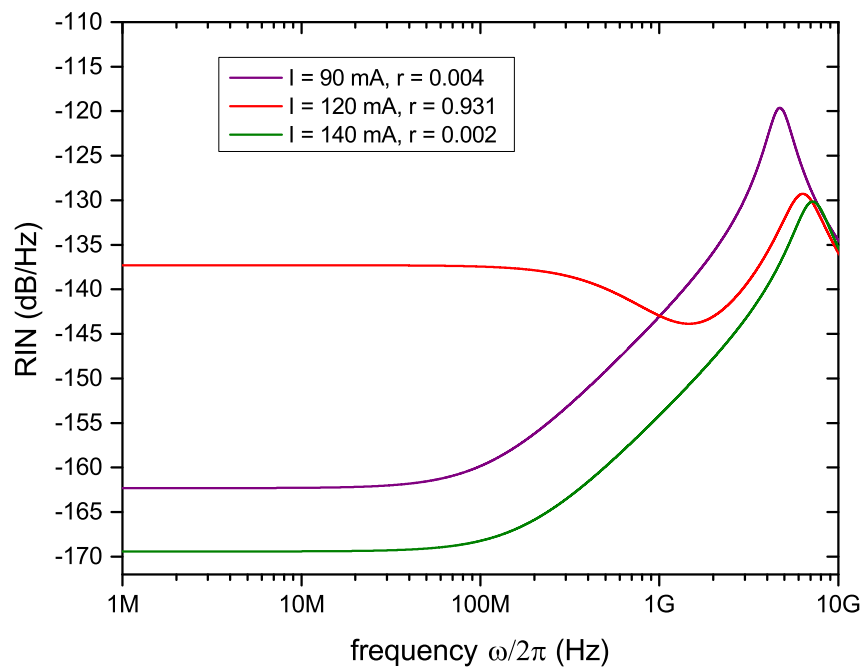

Fig. 6. Variation of the dual-mode RIN for different values of $r$ and $I$ similar to the experimental conditions. Here $D-B=10^{4} \mathrm{~s}^{-1}$ and $\Delta=0.87$.

to the observed phenomenon. Finally, this study reveals that InGaN laser diodes can manifest strong intensity dynamics that can even be detrimental for specific purposes. Nowadays, commercially available monolithic GaN lasers are limited to edge emitting devices. The multimode nature of those lasers strongly favors intensity fluctuations of the output beam as shown in this paper. An attractive solution would be the design of stable single mode lasers. Currently, only external cavity lasers can offer single mode operation in the blue wavelength range hence highlighting the need to develop monolithic single mode InGaN laser diodes.

\section{APPENDIX \\ LANGEVIN FORCES}

To deal with the noise terms, it is assumed that the correlation times of the noise sources are shorter than the photon or carrier lifetimes, i.e., $\gamma^{-1}$ and $\tau_{s}$. This is the socalled Markovian assumption under which, the Langevin terms satisfy the relations [35]

$$
\begin{gathered}
\left\langle F_{i}(t)\right\rangle=0 \\
\left\langle F_{i}(t) F_{j}\left(t^{\prime}\right)\right\rangle=2 D_{i j} \delta\left(t-t^{\prime}\right)
\end{gathered}
$$

where the bracketed terms represent the ensemble average and $D_{i j}$ are the diffusion coefficients which are given as [35]

$$
\begin{gathered}
D_{k k}=R_{s p} \bar{P}_{k}, \quad D_{k l}=0 \\
D_{k N}=-R_{s p} \bar{P}_{k}, \quad D_{N N}=R_{s p} \sum_{k} \bar{P}_{k}+\bar{N} / \tau_{s}
\end{gathered}
$$

where $k=1,2$ and $\bar{N}$ is the steady-state value of the carrier number.

\section{ACKNOWLEDGMENT}

The present work is supported under project Deep Blue by the Region Bretagne (contract $\mathrm{N}^{\circ}$ 16008022) and the European Regional Development Fund (contract N ${ }^{\circ}$ EU000181). 
The authors would like to thank Denis Martin, Jean-François Carlin and Eric Feltin for the growth of the samples and FC équipement for the loan of an optical spectrum analyzer.

\section{REFERENCES}

[1] S. Nakamura, "Characteristics Of Room Temperature-CW Operated InGaN Multi-Quantum-Well-Structure Laser Diodes," MRS Internet Journal of Nitride Semiconductor Research, vol. 2, no. June 2014, p. e5, 1997.

[2] E. Walker, A. Dvornikov, K. Coblentz, and P. Rentzepis, "Terabyte recorded in two-photon 3D disk," Appl. Opt., vol. 47, no. 22, pp. 41334139, Aug 2008.

[3] F. Hanson and S. Radic, "High bandwidth underwater optical communication." Applied Optics, vol. 47, no. 2, pp. 277-283, 2008.

[4] S. Watson, M. Tan, S. P. Najda, P. Perlin, M. Leszczynski, G. Targowski, S. Grzanka, and A. E. Kelly, "Visible light communications using a directly modulated $422 \mathrm{~nm}$ GaN laser diode," Opt. Lett., vol. 38, no. 19, pp. 3792-3794, Oct 2013.

[5] Y.-C. Chi, D.-H. Hsieh, C.-T. Tsai, H.-Y. Chen, H.-C. Kuo, and G.R. Lin, "450-nm GaN laser diode enables high-speed visible light communication with 9-Gbps qam-ofdm," Optics Express, vol. 23, no. 10, pp. $13051-13059,2015$.

[6] X. Zeng and D. L. Boiko, "1 / f noise in external-cavity InGaN diode laser at $420 \mathrm{~nm}$ wavelength for atomic spectroscopy," Optics Letters, vol. 39 , no. 6, pp. 1685-1688, 2014.

[7] H. Leinen, D. Glässner, H. Metcalf, R. Wynands, D. Haubrich, and D. Meschede, "GaN blue diode lasers: a spectroscopist's view," Applied Physics B: Lasers and Optics, vol. 70, no. 4, pp. 567-571, Apr. 2000.

[8] M. G. Allen, "Diode laser absorption sensors for gas-dynamic and combustion flows," Measurement Science and Technology, vol. 9, no. 4, p. $545,1998$.

[9] U. Strau $\beta$, S. Brüninghoff, M. Schillgalies, C. Vierheilig, N. Gmeinwieser, V. Kümmler, G. Brüderl, S. Lutgen, A. Avramescu, D. Queren et al., "True-blue InGaN laser for pico size projectors," in Integrated Optoelectronic Devices 2008. International Society for Optics and Photonics, 2008, pp. 689417-689417.

[10] J. J. Wierer, J. Y. Tsao, and D. S. Sizov, "Comparison between blue lasers and light-emitting diodes for future solid-state lighting," Laser \& Photonics Reviews, vol. 7, no. 6, pp. 963-993, 2013.

[11] F. Arecchi, G. Lippi, G. Puccioni, and J. Tredicce, "Deterministic chaos in laser with injected signal," Optics Communications, vol. 51, no. 5, pp. 308-314, 1984.

[12] G. Acket, D. Lenstra, A. D. Boef, and B. Verbeek, "The influence of feedback intensity on longitudinal mode properties and optical noise in index-guided semiconductor lasers," IEEE Journal of Quantum Electronics, vol. 20, no. 10, pp. 1163-1169, 1984.

[13] I. Joindot, "Measurements of relative intensity noise (RIN) in semiconductor lasers," Journal de Physique III, vol. 2, pp. 1591-1603, 1992.

[14] K. Petermann, Laser diode modulation and noise. Springer Science \& Business Media, Berlin, 1991, vol. 3.

[15] U. T. Schwarz, W. Wegscheider, A. Lell, and V. Haerle, "Nitridebased in-plane laser diodes with vertical currrent path," in Integrated Optoelectronic Devices 2004. International Society for Optics and Photonics, 2004, pp. 267-277.

[16] V. Laino, F. Roemer, B. Witzigmann, C. Lauterbach, U. T. Schwarz, C. Rumbolz, M. O. Schillgalies, M. Furitsch, A. Lell, and V. Härle, "Substrate modes of (Al,In)GaN semiconductor laser diodes on $\mathrm{SiC}$ and GaN substrates," IEEE Journal of Quantum Electronics, vol. 43, no. 1, pp. 16-24, 2007.

[17] T. Meyer, H. Braun, U. T. Schwarz, S. Tautz, M. Schillgalies, S. Lutgen, and U. Strauss, "Spectral dynamics of $405 \mathrm{~nm}$ (Al,In)GaN laser diodes grown on $\mathrm{GaN}$ and $\mathrm{SiC}$ substrate." Optics Express, vol. 16, no. 10, pp. 6833-6845, 2008.

[18] H. Braun, H.-M. Solowan, D. Scholz, T. Meyer, U. T. Schwarz, S. Bruninghoff, A. Lell, and U. Strauß, "Lateral and longitudinal mode pattern of broad ridge $405 \mathrm{~nm}$ (Al, In) GaN laser diodes," Journal of Applied Physics, vol. 103, no. 7, p. 073102, 2008.

[19] I. V. Smetanin and P. P. Vasilev, "Enhanced longitudinal mode spacing in blue-violet InGaN semiconductor lasers," Applied Physics Letters, vol. 100, no. 4, p. 041113, 2012.

[20] T. Weig, T. Hager, G. Brüderl, U. Strauss, and U. T. Schwarz, "Longitudinal mode competition and mode clustering in (Al,In)GaN laser diodes," Optics Express, vol. 22, no. 22, p. 27489, 2014.
[21] J. Dorsaz, A. Castiglia, G. Cosendey, E. Feltin, M. Rossetti, M. Duelk, C. Velez, J.-F. Carlin, and N. Grandjean, "AlGaN-Free Blue III-Nitride Laser Diodes Grown on $c$-Plane GaN Substrates," Applied Physics Express, vol. 3, no. 9, p. 092102, Sep. 2010.

[22] M. Cox, N. Copner, and B. Williams, "High sensitivity precision relative intensity noise calibration standard using low noise reference laser source," IET Science, Measurement and Technology, vol. 145, no. 4, pp. $163-165,1998$.

[23] S. Nakamura, M. Senoh, S.-i. Nagahama, N. Iwasa, T. Yamada, T. Matsushita, Y. Sugimoto, and H. Kiyoku, "Room-temperature continuouswave operation of InGaN multi-quantum-well structure laser diodes," Applied Physics Letters, vol. 69, no. 26, p. 4056, 1996.

[24] H. Jiang and J. Lin, "Mode spacing anomaly in InGaN blue lasers," Applied Physics Letters, vol. 74, no. 8, pp. 1066-1068, 1999.

[25] C. Eichler, S. S. Schad, F. Scholz, D. Hofstetter, S. Miller, A. Weimar, A. Lell, and V. Härle, "Observation of temperature-independent longitudinal-mode patterns in violet-blue InGaN-based laser diodes," IEEE Photonics Technology Letters, vol. 17, no. 9, pp. 1782-1784, Sept 2005.

[26] G. Ropars, A. Le Floch, and G. P. Agrawal, "Spectral and spatial dynamics in InGaN blue-violet lasers," Applied Physics Letters, vol. 89, no. 24, p. 241128, 2006.

[27] Y. Varshni, "Temperature dependence of the energy gap in semiconductors," Physica, vol. 34, no. 149, pp. 149-154, 1967.

[28] M. S. Romadhon, A. Aljalal, W. Al-Basheer, and K. Gasmi, "Longitudinal modes evolution of a GaN-based blue laser diode," Optics \& Laser Technology, vol. 70, pp. 59-62, 2015.

[29] M. Yamada, "Theory of mode competition noise in semiconductor injection lasers," IEEE Journal of Quantum Electronics, vol. 22, no. 7, pp. 1052-1059, Jul 1986.

[30] M. Ohtsu, Y. Otsuka, and Y. Teramachi, "Precise measurements and computer simulations of mode-hopping phenomena in semiconductor lasers," Applied Physics Letters, vol. 46, no. 2, pp. 108-110, 1985.

[31] M. Ahmed and M. Yamada, "Influence of instantaneous mode competition on the dynamics of semiconductor lasers," Quantum Electronics, IEEE Journal of, vol. 38, no. 6, pp. 682-693, 2002.

[32] S. Mikroulis, I. Stamataki, D. Alexandropoulos, M. Hamacher, E. Roditi, and D. Syvridis, "Low-frequency relative intensity noise due to nonlinear mode competition in InGaAsP-InP microring lasers," IEEE Photonics Technology Letters, vol. 18, no. 17-20, pp. 1895-1897, 2006.

[33] E. Lacot and F. Stoeckel, "Nonlinear mode coupling in a microchip laser," Journal of the Optical Society of America B, vol. 13, no. 9, pp. 2034-2040, 1996.

[34] M. Wichmann, G. Town, J. Quante, M. Gaafar, A. Rahimi-Iman, W. Stolz, S. W. Koch, and M. Koch, "Antiphase noise dynamics in a dual-wavelength vertical-external-cavity surface-emitting laser," IEEE Photonics Technology Letters, vol. 27, no. 19, pp. 2039-2042, 2015.

[35] G. P. Agrawal, "Mode-partition noise and intensity correlation in a twomode semiconductor laser," Phys. Rev. A, vol. 37, no. 7, pp. 2488-2494, 1988.

[36] — - "Population pulsations and nondegenerate four-wave mixing in semiconductor lasers and amplifiers," Journal of the Optical Society of America B, vol. 5, no. 1, pp. 147-159, 1988.

[37] W. G. Scheibenzuber, U. T. Schwarz, L. Sulmoni, J. Dorsaz, J.-F. Carlin, and N. Grandjean, "Recombination coefficients of GaN-based laser diodes," J. Appl. Phys., vol. 109, p. 093106, 2011. 